\title{
Calculation of solid particle trajectory inside electrical separator based on measured values
}

\author{
Milan Belik \\ Department of power engineering and ecology \\ University of West Bohemia \\ Pilsen, Czech Republic \\ belik4@kee.zcu.cz
}

\begin{abstract}
This article discusses application of data acquisition device National Instruments NI PCI-6221 for electrical separator low cost monitoring. Results can be applicable for air protection and air pollution prevention. Measured basic electrical and environmental values are logged into a file. Further the data serve as input for calculation of solid particles trajectory inside the separator. Influence of particular quantities onto the separator operation and particle trajectory calculation is discussed. Theoretical and technical limits of used device for high voltage measurements are explained in the first part. Main part of the paper deals with calculation of the trajectory and implementation in LabVIEW environment. Influence of particular physical parameters is elaborated. Presented ideas are generalized for more complex system in last chapter.
\end{abstract}

Keywords- measurement and monitoring in air protection; electrical separator; solid particle trajectory; data acquisition; LabVIEW

\section{INTRODUCTION}

The main task of this paper is to discuss calculations of solid particle trajectory inside the electrical separator. Computation is based on data measured on the separator and its environment with low cost data acquisition system using National Instruments NI PCI 6221 hardware and LabVIEW interface. General multifunctional hardware and universal software platform regards the implementation into more complex automated system serving for particular task in air protection and air pollution monitoring [1].

Electrical separators are commonly used to separate solid particles originated from technological processes in industry and above during fossil fuels burning in power engineering. The operation of the separators depends on electrostatic field between high voltage charging electrode and grounded collecting electrode. These separators are dominant in all applications with high volumetric flow and high gas temperature [1].

The paper was supported by SGS-2018-023.
Several specific conditions must be fulfilled to achieve high separation efficiency. External force affecting the separation speed at collecting electrode is the Coulomb force. It depends on the gaseous environment inside the separator, flow speed, electrodes conditions and features of the particles.

Measurement of the electric field and physical conditions inside the separator is the first task of this project. General multipurpose hardware is adopted for several various measurements using specific implementation of one common service routine composed in LabVIEW [2].

Measured data and setup of particle parameters are the input for the proper calculation of the trajectory inside the separator using another LabVIEW code.

Presented system should be a complex low cost alternative to usage of commercial single purpose analyzers. LabVIEW program substitutes simulations in general scientific environments such as MATLAB or Mathematica [2, 3].

Technical limitations of used hardware are discussed in detail in chapter II. Next chapter describes usage of the hardware for measurements of electric separator. VA characteristic of the separator is deeply explained on several examples. Following chapter deals with data acquisition. DAQ process is implemented in LabVIEW. Results are presented on a set of measured VA characteristics. Finally chapter IV explains the calculation of the trajectory inside the separator. The algorithm is applied in LabVIEW environment. All results are summarized and discussed in final chapter.

\section{TeChnical Limitations Of DeVELOPED DATA ACQUISITION SYSTEM}

The developed data acquisition system is based on general multipurpose National Instruments NI-PCI 6221 hardware. It offers low-cost reliable DAQ capabilities. Enclosed driver for LabVIEW platform allows simple configuration for all measurement operations [4].

NI-PCI $6221 \mathrm{I} / \mathrm{O}$ device disposes of 16 analog input channels, 2 analog output channels and 24 digital input / output channels. The sampling frequency is $250 \mathrm{kSs}^{-1}$. Analog channels contain 16 bit AD transducers. Input voltage range is $\pm 10 \mathrm{~V}$. Device has implemented two 32 bit counters / timers, but they are 
not applied in this application. The voltage range of the device does not allow direct measurements necessary for the electrical separators. This issue can be simply solved with coupling resistances or with transducers.

Terminal side of this DAQ chain is standard 68 pin connector block CB-68LP. The block allows connection of any external equipment depending on measurement purpose [4].

\section{MEASUREMENTS ON ELECTRIC SEPARATOR}

Input analog channels of NI PCI-6221can be wired either as reference zero or as floating zero. Voltage range allows measurement of both polarities, but the bounds of the range limit direct measurements.

High voltages necessary for correct function of electric separators must be measured indirectly. This application can contain either voltage divider or galvanic separation.

Fig. 1 demonstrates voltage divider wired to the terminal block for high voltage measurements on electric separator. Input channels can be reconfigured in DAQ Assistant or directly in features of NI PCI6221 in block diagram. The measurement requires isolated zero. Fig. 1 shows the usage of DAQ Assistant for configuration.

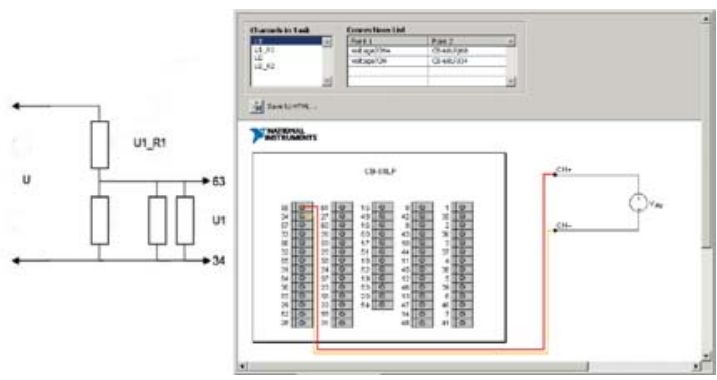

Figure 1. Sample connection and DAQ assist configuration

Fig. 2 presents main segment of block diagram in LabVIEW for high voltage measurements on the electric separator. 2 channels are capable to acquisite voltage upto $100 \mathrm{kV}$. Maximum voltage between the electrodes of the separator is below this limit, so the full set of VA characteristics can be measured. Block diagram for measurements of gas temperature, gas pressure and humidity uses similar structure.

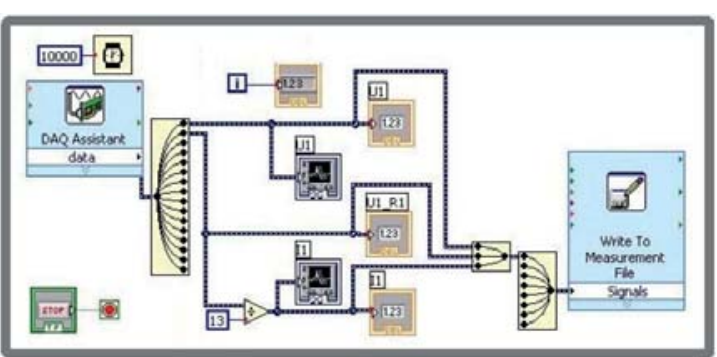

Figure 2. Block diagram for voltage measurement of the separator

These measured data are further analyzed in sample service utility. Front panel of the utility is presented on Fig. 3.

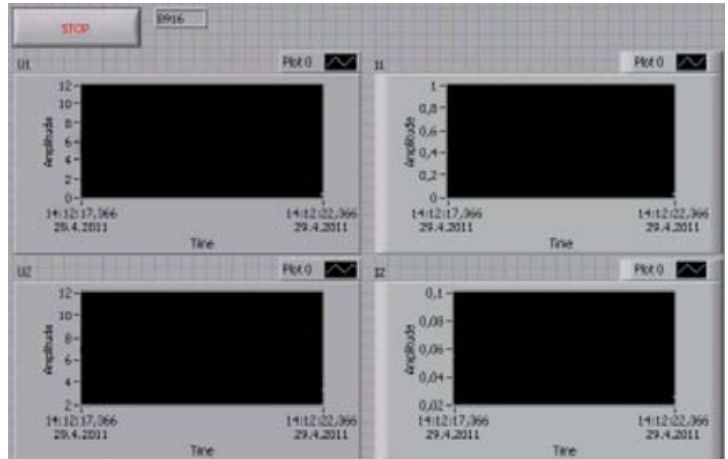

Figure 3. Front panel for voltage measurement of the separator

The values and the shape of the electrostatic field play very important role in the electric separator. The field impacts the separation process at first directly, because it generates the external force acting the particles. Secondly it influences the separation indirectly, because it causes collision ionization and thus charging of the particles. Both steps follow and affect each another.

Field in chamber separator (1) depends on distance between the electrodes $\mathrm{h}$, geometrical order factor $\mathrm{f}$, stereometric shape distribution factor X and voltage $\mathrm{U}$.

$$
E=\frac{\pi U}{2 h f} \mathrm{X}
$$

If the intensity $E$ exceeds crtical value $E_{k r i t}$, collision ionization (corona) occurs. Free electrons and cations of the gas are generated during this process. The majority of industrial separators use high voltage electrodes of negative polarity, so the corona is also negative. Generated electrons move along field lines, earn high speed and thus energy. It they hit onto neutral molecules of the gas, the cause secondary ionization. In areas with lower intensity (and lower electron speed) electrons have enough time to diffuse into neutral molecules. It further causes secondary ionization.

Empiric equation (2) describes critical intensity $\mathrm{E}_{\text {krit }}$ in ionization area. Constants $\mathrm{K}_{1}, \mathrm{~K}_{2}$ depend on gas composition and configuration of electrodes. Coefficient $\beta$ is gas density factor and $r$ represents the radius of high voltage electrode.

$$
E_{k r i t}=K_{1} \beta_{\rho}\left(1+\frac{K_{2}}{\sqrt{\beta_{\rho} r_{i}}}\right)
$$

The size of the ionization area can be calculated from equation (3).

$$
\mathrm{E}>\mathrm{E}_{\mathrm{krit}}
$$

If the voltage on the high voltage electrode is higher than $\mathrm{E}_{\text {krit, }}$ ionization occurs and the current starts to flow between electrodes. The value of $E_{\text {krit }}$ depends on ambient conditions. Fig. 4 shows the dependency of measured VA characteristic on the gas pressure, while Fig. 5 demonstrates dependency on the gas temperature. 


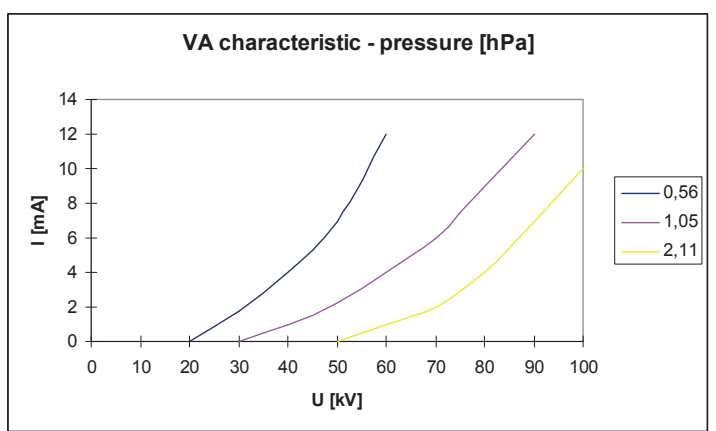

Figure 4. VA characteristic dependency on gas pressure

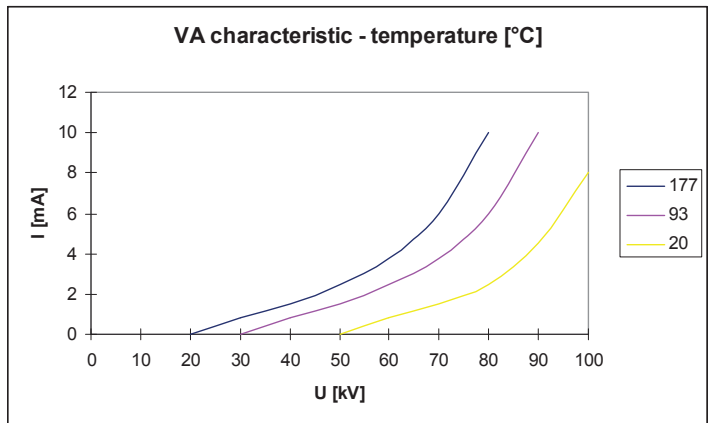

Figure 5. VA characteristic dependency on gas temperature

Analogous dependency can be found for variable humidity as presented on Fig. 6.

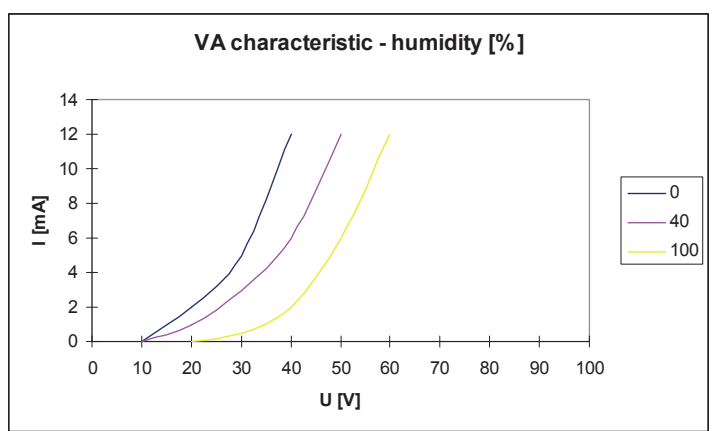

Figure 6. VA characteristic dependency on humidity

Gas chemical composition, diameter of high voltage electrode, stepping of both electrodes, particles concentration and electrodes pollution also affect the VA characteristic in similar way.

\section{Sequential Data AcQuisition ON ELECTRICAL SEPARATORS}

Used NI PCI-6221device disposes not only of analog input channels but also of 24 digital input / output channels. These digital channels can be used for variable switching operations during data acquisition.

This feature allows utilization of 24 different wirings during automated measurements on electrical separators. Output of these digital channels is TTL compatible, so that direct switching in this application is not possible. To avoid damage of the device 12 pieces of RS232 Serial Control for DC 5V 2-Channel Relays can be used.

Original idea was to use single separate channel for each predesigned wiring of particular sensors. First channel (D0) is wired to no load and can be used for shortcut measurement. Channels D1 - D22 can contain 22 different particular sensors for 22 points of the VA characteristic. Last channel (D23) is wired as open and can be used for open circuit voltage measurement.

Actual number of utilized channels depends on number of measuring points and sensors. Fig. 7 shows sample block scheme with switching relays (RS232 Serial Control for DC 5V 2-Channel Relay).

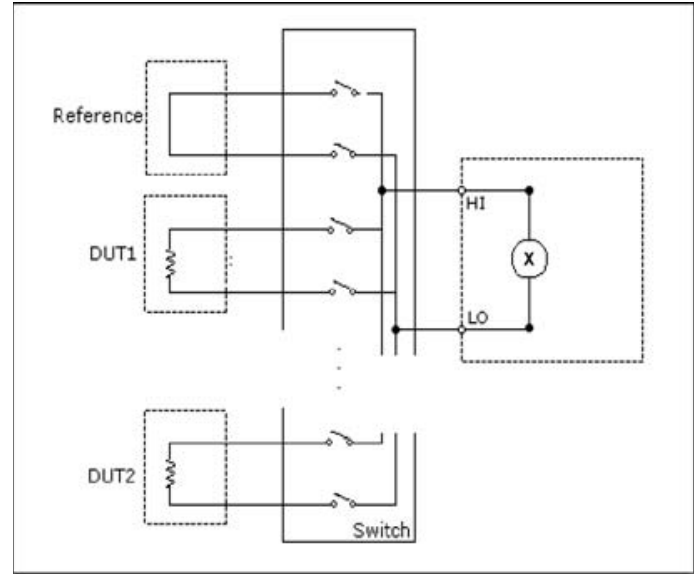

Figure 7. Multi channel sequential measurements

The data acquisition calls subroutine (sub-VI) with analogical scheme to Fig. 2. The sub-VI is controlled from flat sequence structure. The structure triggers predefined sequence of digital channels wired to relays (RS232 Serial Control for DC 5V 2-Channel Relay). Sample inset of this routine in general block diagram in LabVIEW is presented on Fig. 8.

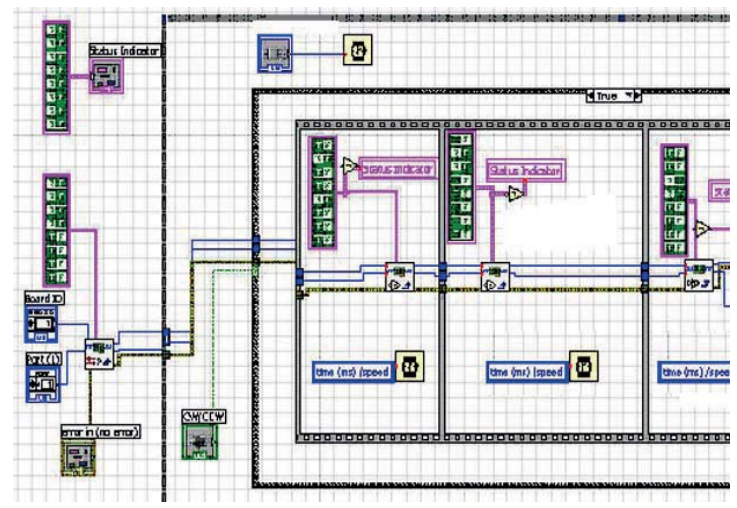

Figure 8. Block diagram for flat sequence triggering

Fig. 9 shows sample of 5 VA characteristics measured at constant conditions (gas pressure: $0,948 \mathrm{hPa}$, gas temperature: $168^{\circ} \mathrm{C}$, humidity: $18 \%$ ). Particular characteristics were measured in sequence with switching interval $10 \mathrm{~min}$. 


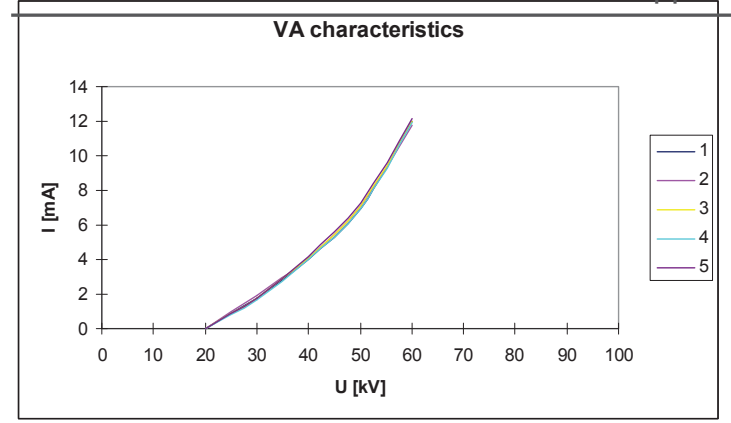

Figure 9. Sample measured VA characteristics

\section{Calculation Of Solid Particle}

\section{TRAJECTORY INSIDE THE ElECTRICAL SEPARATOR}

Separation process in two-phase systems consisting from a gas and solid particles is based on the particles transition from the carrying gas onto separation surface. The transition process is the result of acting mass forces (gravitational, inertial, centrifugal, magnetic) and surface forces (aerodynamic resistance, molecular, electrical).

Equation of motion composition appears from the second Newton law as demonstrated on Fig. 10. Solid particle with speed vector $u$ is situated in carrying gas with speed vector $v$. The particle is than circumfluented with the relative speed represented with vector $\mathrm{v}_{\mathrm{r}}$.

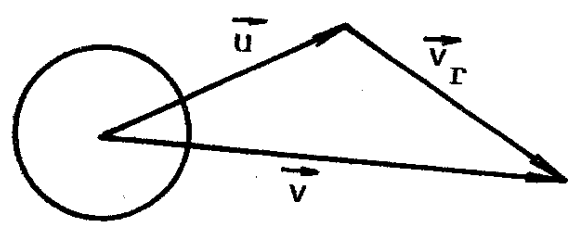

Figure 10. Kinematic of solid particle in carrying gas

Inertial force (4) can be expressed from the speed $u$ and particle mass $\mathrm{M}$. The mass depends on particle density $\rho$ and particle diameter a.

$$
\vec{F}_{1}=M_{\check{c}} \frac{d \vec{u}}{d t}=\frac{\pi a^{3}}{6} \rho_{\check{c}} \frac{d \vec{u}}{d t}
$$

While the particle is in motion in viscous environment, certain volume of the environment is pulled behind the particle. The volume is estimated to be one half of the particle volume and represents mass $\mathrm{M}_{\mathrm{p}}$. Inertial force of the assigned volume (5) depends on the relative speed equal to vector difference between $\mathrm{u}$ and $\mathrm{v}$.

$$
\vec{F}_{2}=M_{p} \frac{d(\vec{u}-\vec{v})}{d t}=\frac{\pi a^{3}}{12} \rho\left(\frac{d \vec{u}}{d t}-\frac{d \vec{v}}{d t}\right)
$$

Aerodynamic resistance of the particle (6) depends on relative speed $\mathrm{v}_{\mathrm{r}}$, density of the environment $\rho$ and resistance coefficient $\xi$.

$$
\vec{F}_{\tau}=\xi \frac{\pi a^{2}}{4} \frac{|\vec{v}-\vec{u}|(\vec{v}-\vec{u})}{2} \rho
$$

If the particle exists in environment with variable static pressure, buoyancy force (7) takes effect. It depends on the pressure gradient $p$.

$$
\vec{F}_{y}=-V_{\dot{c}} \vec{\nabla} p
$$

Typical calculation of the trajectory is based on equality of all these forces. Final representation (8) can be derived from this condition respecting sum of all other external forces $\mathrm{F}_{\mathrm{e}}$.

$$
\begin{aligned}
& \frac{\pi a^{3}}{6} \rho_{\check{c}} \frac{d \vec{u}}{d t}+\frac{\pi a^{3}}{12} \rho\left(\frac{d \vec{u}}{d t}-\frac{d \vec{v}}{d t}\right)= \\
& =\xi \frac{\pi a^{2}}{4} \frac{\vec{v}-\vec{u}((\vec{v}-\vec{u})}{2} \rho-\frac{\pi a^{3}}{6} \vec{\nabla} p+\vec{F}_{e}
\end{aligned}
$$

Equation of motion application in LabVIEW routine shows Fig. 11. Numeric controls in the sample part of wire diagram represent inputs for particular forces $\left(\mathrm{F}_{1}, \mathrm{~F}_{2}, \mathrm{~F}_{\tau}, \mathrm{F}_{\mathrm{v}}\right.$ and $\left.\mathrm{F}_{\mathrm{e}}\right)$.

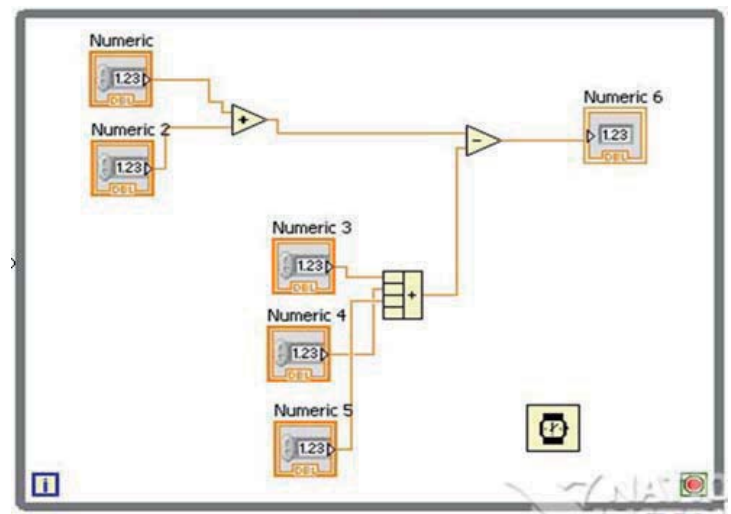

Figure 11. Block diagram for the equation of motion calculation

Simulations based on data acquisited according to Fig. 8 and calculated according to Fig. 11 allow draw the trajectory of the solid particle in electrical separator. Fig. 12 demonstrates sample result of this simulation.

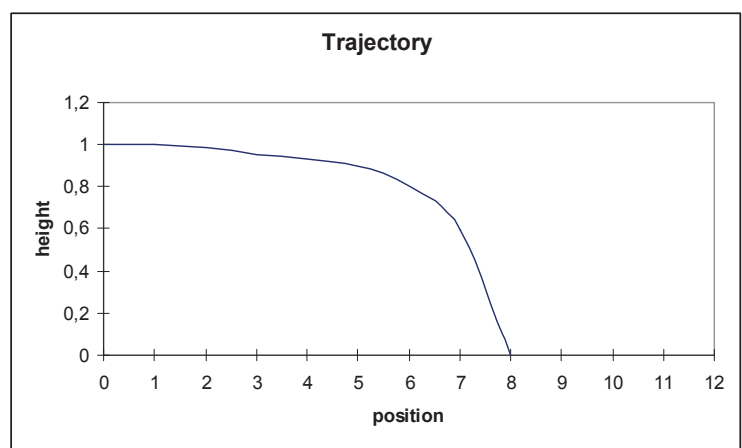

Figure 12. Sample calculated trajectory 


\section{RESULTS AND CONCLUSIONS}

Presented measurements and simulations demonstrate capability of data acquisition device NI PCI 6221 for purposes in air protection applications.

The system benefits from universal architecture capable for data acquisition and also for controlling of the external equipment. Relatively low price is also significant advantage. PCI architecture guarantees good compatibility and easy implementation to more complex systems.

This application extended the set of developed NI PCI 6221 applications for power engineering. It can be used either to monitor the operation of electric separator in the industry or to control air pollution in environmental protection tasks.

Fig. 12 proves that the result of the entire process is a smooth curve. It indicates relevant accuracy either of data acquisition or of the equation of motion calculations. The accuracy of data acquisition is limited with technical limits of NI PCI-6221device. 16 bit $\mathrm{AD}$ converters of 16 analog channels and sampling frequency $250 \mathrm{kSs}^{-1}$ are sufficient for this task. Accuracy of the calculation depends on data representation. Double precision representation brings sufficient accuracy with reasonable hardware requirements.

Calculated trajectory complies with theoretical expectations, but deeper research should be executed on the last phase of the motion, when the particle is captured on the separation surface. Rebound of the particle can occur in dependency on the material and physical features of the particle and on conditions in the separator. This new particle position is the starting point for another run of described algorithm. The future task will be evaluation of this process and finding of particle new steady position.

\section{REFERENCES}

[1] Hemerka, J., Zaklady ochrany ovzdusi, CVUT, Praha, 2008.

[2] Golkar, M., A., Electric power quality: types and measurements, Proceedings of the DRPT, Hong Kong, 2004.

[3] Spanik, P., Hargas, L., Hrianka, M., Kozehuba, I., Application of Virtual Instrumentation LabVIEW for Power Electronic System Analysis, Power Electronics and Motion Control Conference, Portoroz, 2009.

[4] Device specification NI 6221, National Instruments, 2015.

[5] Tarasiuk, T., Pilat, A., Impact of sampling frequency on accuracy of unbalance factor measurement by DFT, Instrumentation and Measurement Technology Conference, Pisa, 2015.

[6] C.A.8335 Qualistar Plus, Blue Panter, Praha, 2012. 